\title{
Gerador de Padrões para Representação de Sinais Ópticos com Presença de Impulsividade
}

\author{
A. Aldano e R. Coelho
}

\begin{abstract}
Resumo-Um novo método de geração de sinais ópticos com padrão baseado na família de distribuições alpha-stable $(\alpha-S)$ é apresentado neste trabalho. O padrão $\alpha-S$ é considerado mais apropriado para a aproximação de sinais e ruídos reais do que a distribuição Gaussiana. Os resultados de validação obtidos pela solução, proposta para implementação em hardware, demonstraram eficiência na representação do padrão $\alpha-S$ e dos indíces de impulsividade. Eles também evidenciam a relação entre os graus de impulsividade e a cauda-pesada ou heavy-tail distribution (HTD).
\end{abstract}

Palavras-Chave-geração sinais ópticos, impulsividade, padrões alpha-stable e de cauda-pesada.

Abstract-This paper presents a novel optical signal generation method with alpha-stable $(\alpha-S)$ pattern. The $\alpha-S$ is considered more suitable for the representation of real signals and noise than the Gaussian distribution. The solution is presented and examined for hardware samples generation. The experimental results showed that the proposed generation method achieved the $\alpha-S$ pattern representation and the impulse behavior represented by the $\alpha$ values. The results also show the relationship between the $\alpha$ (impulse) and the heavy-tail distribution (HTD) parameters.

Keywords-optical signal generation, $\alpha$-stable- pattern, impulse, HTD.

\section{INTRODUÇÃO}

A redução do custo dos sistemas de comunicações ópticos, associada à importantes características tais como a confiabilidade do meio de transmissão, a baixa perda de informação e a imunidade a ruídos eletromagnéticos, vêm popularizando a sua implantação em larga escala [1] [2] [3]. Sistemas residenciais como o FTTH (fiber-to-the-home), já são uma realidade e também responsáveis pelo incremento de acessos em banda larga à Internet em alguns países. No Japão, acredita-se que o número de acessos, via FTTH, passará de 2,8 milhões (1995) para 30 milhões de acessos até 2010 [4].

Para a obtenção do melhor dimensionamento, avaliação de desempenho e testes experimentais dos sistemas de comunicações ópticos, é fundamental o emprego de geradores de sinais e ruídos com diferentes padrões ou distribuições. Geradores desenvolvidos em hardware demostram ter processamento mais rápido e maior precisão na geração de amostras [5] [6]. A maioria das soluções de geração de amostras apresentada na literatura, é desenvolvida para o padrão Gaussiano. Estas propostas são baseadas no clássico método Box-Müller [7] e no teorema do limite central.

Os autores estão no Departamento de Engenharia Elétrica do Instituto Militar de Engenharia (IME), Laboratório de Comunicações e Sistemas Ópticos (LaRSO). Email: \{aldano,coelho\}@ime.eb.br. Este trabalho foi parcialmente suportado pelo CNPq e pelo University Research Grant da CISCO/USA.
Este trabalho apresenta um novo método de geração de sinais com padrão baseado na família de distribuições $\alpha$ stable para implementação em hardware. O gerador foi implementado e testado em arquitetura FPGA (field programmable gate array) da Altera utilizando o kit de desenvolvimento Stratix EP1S25 e o ambiente Quartus II para Linux. O padrão $\alpha$-S é mais apropriado para a aproximação de sinais e ruídos reais [8] [9] [10] devido a representação dos diferentes indíces de impulsividade $(\alpha)$. Variáveis aleatórias (V.A.s) com distribuição $\alpha-S$ são também denominadas na literatura como não-Gaussianas. Alguns casos particulares desta família são as distribuições Gaussiana e Cauchy com índices de impulsividade definidos por $\alpha=2$ e $\alpha=1$, respectivamente.

Processos estocásticos $(X(t))$ com distribuição de caudapesada, ou seja, $P[X(t)>x] \cong c x^{-\alpha}, x \rightarrow \infty$, onde $c$ uma constante positiva e $\alpha \in(0,2)$, podem apresentar alto grau de impulsividade ou variância infinita [11] [9]. Esta impulsividade pode ser atribuída ao lento decaimento da cauda em comparação à distribuição Gaussiana. A impulsividade de sinais com cauda-pesada, é mantida mesmo após a agregação de inúmeras ordens do processo ou multiplexação de sinais. Quanto menor o valor de $\alpha$, mais pesada ou longa será a cauda da distribuição.

Nos experimentos as amostras geradas no meio eletrônico (FPGA), são posteriormente codificadas (bit-serial) e moduladas no comprimento de onda 1550,92 nm de uma fonte laser operando em frequiência de $622 \mathrm{MHz}\left(f_{0}\right)$. Assim, pulsos de luz foram gerados a cada $T$ segundos $\left(T=1 / f_{0}\right)$ e o sinal óptico é obtido segundo o padrão $\alpha-S$. Após a geração e a transmissão ópticas, os bits são re-convertidos para o meio eletrônico onde é realizada a validação do padrão e as medidas de desempenho pertinentes.

Os resultados experimentais do gerador de padrões $\alpha-S$ foram obtidos considerando-se diferentes indíces de impulsividade. Um grande desafio dos estudos [8] [12] baseados em padrões $\alpha$-S, concerne a complexidade da estimação de seus parâmetros básicos. Esta complexidade se deve justamente ao aspecto impulsivo presente nas amostras, principalmente para valores $\alpha<1,7$. Segundo [13], a probabilidade de ocorrência de erros de precisão, de até $100 \%$, na estimação dos parâmetros e medidas em amostras com padrão $\alpha-S$, não é desprezível $(\approx 0,03)$. Os resultados de validação do gerador de padrões proposto neste trabalho, envolvem a estimação de seus parâmetros básicos, as curvas das distribuições de cauda pesada obtidas bem como os parâmetros de desempenho da solução FPGA (uso de memória, elementos lógicos, clock). Os resultados obtidos com a geração em hardware foram comparados com os valores resultantes da geração de amostras $\alpha-S$ segundo a família de distribuição definida por Lévy 
realizada em programação $\mathrm{C}++$. Esta solução é aqui denotada por software (SW) ou teórica.

O restante deste trabalho está organizado da seguinte forma: $\mathrm{Na}$ seção II são apresentadas as principais definiçõos, a representação e a estimação dos indíces de impulsividade e demais parâmetros do padrão $\alpha-S$. A proposta de geração de sinais com padrão $\alpha-S$ simétrico $(S-\alpha-S)$ é apresentada na seção III. Na seção IV, observa-se a arquitetura do bloco FPGA do padrão $S-\alpha-S$. Os principais resultados de validação e dos testes experimentais, com os sinais ópticos, são discutidos na seção VI. Finalmente, a seção VII apresenta as conclusões deste trabalho.

\section{IMPUlSIVIDADE: DEFINIÇÃO, REPRESENTAÇÃO E ESTIMAÇÃO}

A família de distribuições $\alpha-S$ definem e modelam fenômenos com característica de impulsividade e caudapesada. O termo impulsividade caracteriza um certo grau de estabilidade, definido por $\alpha$, em contraponto a nãoimpulsividade da distribuição Gaussiana $(\alpha=2)$. Esta família foi definida por Paul Lévy [14] em 1925 e, deste então, vêm sendo aplicada à caracterização de fenômenos com presença de impulsividade observados nas mais diferentes áreas tais como economia, hidrologia, biologia, física, entre outras.

\section{A. Definição}

As principais características da família de distribuições $\alpha-S$ são:

- Propriedade da estabilidade: Considerando as V.A.s $X$, $X_{1}$ e $X_{2}$ independentes com distribuição $S-\alpha-S$, então existem $a$ e $b$ que satisfazem

$$
c_{1} X_{1}+c_{2} X_{2} \stackrel{d}{\approx} a X+b
$$

onde $c_{1}, c_{2}, a$ e $b$ são constantes e $\stackrel{d}{\approx}$ significa semelhança em distribuição.

- Teorema do limite central generalizado: Sejam $a_{n}$ e $b_{n}$ constantes reais positivas. $X$ é uma V.A. $\alpha-S$ se e somente se, $X$ é o limite em distribuição da soma $S_{n}=\frac{X_{1}+X_{2}+\ldots+X_{n}}{a_{n}}-b_{n}$ onde $X_{1}, X_{2}, \ldots, X_{n}$ são V.A.s independentes e identicamente distribuídas (i.i.d) e $n \rightarrow \infty$.

A distribuição $\alpha$-S é definida por 4 parâmetros básicos $(\alpha$, $\beta, \mu, \gamma)$ :

- $\alpha$, denominado índice de impulsividade ou expoente da função característica, é definido no intervalo $0 \leq \alpha \leq 2$. Quanto menores os valores de $\alpha$ mais pesada é a cauda da distribuição e mais impulsiva é a seqüência amostral.

- $\beta$ define o grau de assimetria sendo geralmente representado por $-1 \leq \beta \leq 1$. Quando nulo, representa a distribuição $\alpha-\bar{S}$ simétrica $(S-\alpha-S)$ sendo estes os padrões mais abordados na literatura e também neste trabalho. A simetria é definida em torno do parâmetro de localização $(\mu)$.

- $\mu$ é o parâmetro de localização. Ele pode assumir qualquer valor real $(-\infty \leq \mu \leq \infty)$.
- $\gamma$ é o parâmetro de escala ou dispersão $(0 \leq \gamma \leq \infty)$. Este representa o grau de dispersão em torno do parâmetro de localização. Este parâmetro é também definido por alguns autores por $c=\gamma^{\frac{1}{\alpha}}$ e representaria a variância estatística dos sinais.

\section{B. Representação do padrão $\alpha-S$}

Não há expressão fechada para a função densidade de probabilidade (fdp), $f_{X}(x)$, da família de distribuição $\alpha-S$. Sua caracterização é geralmente feita através de sua função característica $(\phi(t))$

$$
\phi(t)=E\left(e^{i t x}\right)=\int_{\Omega} e^{i t x} f_{X}(x) d x .
$$

onde

$$
\phi(t)=\exp \left[i t \mu-|\gamma t|^{\alpha}(1-i \beta \operatorname{sgn}(t) \Phi)\right] .
$$

sendo

$$
\operatorname{sgn}(t)=\left\{\begin{array}{cc}
1, & x>0 \\
0, & x=0 \\
-1, & x<0
\end{array}\right.
$$

e

$$
\Phi=\left\{\begin{array}{cc}
\tan (\alpha \pi / 2), & \alpha \neq 1 \\
-(2 / \pi) \log |t|, & \alpha=1
\end{array}\right.
$$

Assim as fdps do padrão $S-\alpha-S$ podem ser obtidas realizando-se algumas transformações

$$
f_{X}(x)=\frac{1}{2 \pi} \int_{-\infty}^{\infty} e^{-i t x} \phi(t) d t .
$$

Para $\alpha=2$ e $\beta=0$ encontra-se

$$
f_{X}(x)=\frac{1}{\sqrt{4 \pi \gamma}} \exp \left\{-\frac{(x-\mu)^{2}}{4 \gamma}\right\}
$$

que é a distribuição Gaussiana de média $\mu$ e variância $\gamma$. Este é um caso limite da $S-\alpha-S$. Da mesma forma, encontra-se a distribuição de Cauchy para $\alpha=1$ e $\beta=0$ :

$$
f_{X}(x)=\frac{\gamma}{\pi\left[\gamma^{2}+(x-\mu)^{2}\right]}
$$

e Lévy para $\alpha=1 / 2$ e $\beta=1$ :

$$
f_{X}(x)=\sqrt{\frac{\gamma}{2 \pi}} \frac{1}{(x-\mu)^{3 / 2}} \exp \left(-\frac{\gamma}{2(x-\mu)}\right) .
$$

\section{Estimação dos Parâmetros $\alpha, \beta, \mu, \gamma$}

Os principais métodos de estimação dos parâmetros básicos de amostras com padrão $S-\alpha-S$, foram propostos na literatura por McCulloch [15], Kogon [16], Ma [17] e Tsihrintzis [18]. Uma detalhada análise comparativa do desempenho de estimação destes algoritmos é apresentada em [12]. Os autores concluiram que o método proposto por McCulloch, baseado na estatística de ordem fracionária [9] de uma seqüência amostral, é o mais completo e preciso para a estimação dos 4 parâmetros básicos. Além disso eles verificaram que, quando os valores dos métodos de estimação divergiam muito entre si, significava que as amostras não seguiam verdadeiramente uma distribuição $S-\alpha-S$. Contudo, a estimação dos parâmetros de padrões $S-\alpha-S$ ainda é um importante desafio para as 
pesquisas da área de teoria de estimação. Segundo [13], erros de precisão da ordem de $100 \%$, na estimação dos parâmetros podem ocorrer para amostras alta impulsividade ou com $\alpha<$ 1,7. Neste trabalho foi implementado o método de McCulloch para a estimação dos parâmetros das sequiências amostrais obtidas pelo gerador do padrão $S-\alpha-S$ proposto.

\section{Geração Padrão $\alpha$-Stable Simétrico}

Para a geração de amostras em hardware, segundo as fdps $S-\alpha-S$ apresentadas na seção II.B, foi adotado o sistema de transformação proposto por Nolan [19]. O método consiste em aplicar transformações (funções logaritmicas e trigonométricas) sobre um par de V.A.s com distribuição Uniforme de modo a obter o padrão $S-\alpha-S$ desejado. Assim, considere 2 V.A.s uniformes e independentes $u_{1}$ e $u_{2}$ e $W=$ $-\log u_{2} \Theta=\pi\left(u_{1}-1 / 2\right)$. Então pode-se obter uma V.A. $S$ - $\alpha$ - $S Z(\alpha, \beta, 1,0)$ da seguinte forma:

- Para $\beta=0$

$$
Z=\left\{\begin{array}{cc}
\frac{\operatorname{sen}(\alpha \Theta)}{(\cos \Theta)^{1 / \alpha}}\left[\frac{\cos (\alpha-1) \Theta}{W}\right]^{(1-\alpha) / \alpha} & \alpha \neq 1 \\
\tan \Theta & \alpha=1
\end{array}\right.
$$

- $\operatorname{Para} \beta \neq 0$

$$
Z=\left\{\begin{array}{c}
\frac{\operatorname{sen} \alpha\left(\theta_{0}+\Theta\right)}{\left(\cos \alpha \theta_{0} \cos \Theta\right)^{1 / \alpha}}\left[\frac{\cos \left(\alpha \theta_{0}+(\alpha-1) \Theta\right)}{W}\right]^{\frac{1-\alpha}{\alpha}} \\
\frac{2}{\pi}\left[\left(\frac{\pi}{2}+\beta \Theta\right) \tan \Theta-\beta \log \left(\frac{\frac{\pi}{2} W \cos \Theta}{\frac{\pi}{2}+\beta \Theta}\right)\right]
\end{array}\right.
$$

Finalmente, para obtenção de um sinal com padrão $S$ - $\alpha-S$ $X(\alpha, \beta)$ e com dispersão $(\gamma)$ diferente da unidade e localização $(\mu)$ não-nula, prossegue-se à transformação:

$$
X=\left\{\begin{array}{cc}
\gamma Z+\mu, & \alpha \neq 1 \\
\gamma Z+\left(\mu+\beta \frac{2}{\pi} \gamma \log \gamma\right), & \alpha=1
\end{array}\right.
$$

\section{Arquitetura Bloco FPGA de Geração $S-\alpha-S$}

O kit FPGA Stratix adotado no desenvolvimento do gerador de padrões da família $S-\alpha-S$ possui alta densidade de elementos lógicos (aproximadamente 25.000). O Stratix oferece todos os elementos necessário à implementação dos blocos de geração do padrão $S$ - $\alpha$ - $S$ sem comprometer o desempenho (freqüência máxima de operação).Os seguintes elementos digitais, utilizados na implementação do bloco $S$ $\alpha-S$, podem ser destacados:

- multiplicadores embarcados que são elementos que implementam operações de multiplicação direta em hardware:

- 4 blocos de memória RAM totalizando 2 Mbits.

- 10 blocos de DSP (digital signal processors) usados no processador embarcado PMax.

- 6 PLL's (phase-locked loop) utilizados na geração de clock.

- elevado número de circuitos de entrada e saída, facilitando a interconexão dos CIs (circuitos integrados) auxiliares.

A programação da FPGA foi realizada na plataforma de desenvolvimento Quartus II que permite tanto a linguagem de programação de hardware VHDL (Very High Speed Hardware

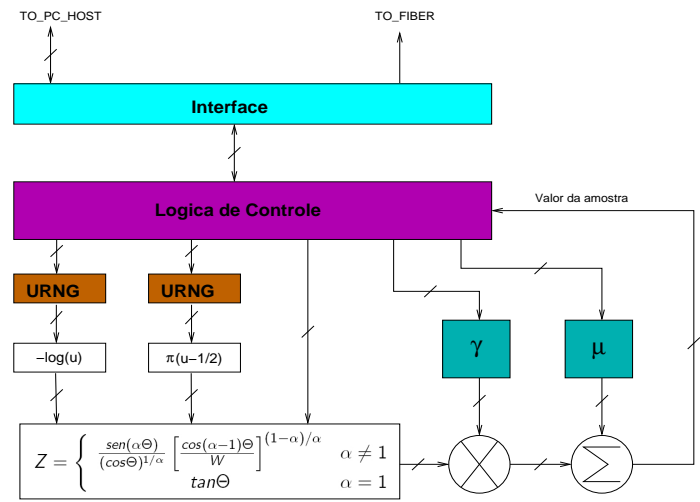

Fig. 1. Arquitetura da geração FPGA de amostras padrão $S-\alpha-S$.

Description Language), quanto as lógicas elementares (por exemplo AND, XOR e OR) que são conectados na forma de blocos esquemáticos. Após as fases de síntese e adequação, a FPGA é fisicamente programada. O Quartus II implementa o que foi previsto para o bloco de geração pelo código do programa, utilizando as lógicas elementares definidas na FPGA. Esta fase é demorada e complexa pois nem sempre é possível implementar uma função diretamente em hardware sem o uso de técnicas especiais (Por ex. lookup-table [5]). Em seguida, é gerado um arquivo binário para configuração da FPGA. Este arquivo é geralmente configurado via porta paralela e permanece assim enquanto houver alimentação da fonte de energia. Neste trabalho, foi utilizado o processador PMax embarcado na FPGA. Este processador é gerado no Quartus II de acordo com as funcionalidades determinadas pelo programador. O conjunto de dados e as instruções do processador PMax é gerado em outra plataforma de programação, o IDE-Nios2. Nesta plataforma é possível escrever programas na linguagem $\mathrm{C} / \mathrm{C}++$, e executá-los diretamente no PMax. A plataforma IDE-Nios2 cria a partir do código em $\mathrm{C} / \mathrm{C}++$, um conjunto de micro-instruções para que o processador PMax possa interpretar. A grande vantagem do emprego deste recurso é também de implementar de forma rápida e precisa, o controle da troca de informações entre os diferentes unidades que compõem a geração dos padrões.

A Fig.1 ilustra a arquitetura do bloco FPGA para geração de sinais com padrão $S$ - $\alpha$ - $S$ proposto neste artigo. O bloco de interface é responsável pela codificação dos sinais para envio ao PC controlador, transmissão pela fibra e ainda por decodificar os comandos advindos do controlador. A lógica de controle gera os sinais do bloco necessários ao controle das unidades operacionais subjacentes.

Os blocos geradores de amostras uniforme (URNGuniform random number generator) estão implementados numa combinação dos métodos LFSR (multiple-bit leapfoward shift register) e CASR (cellular automata shift register) utilizando registradores de 43 bits. O método de geração dos números aleatórios adotado foi proposto por Tkacik em [20]. Os blocos URNG geram amostras de 32 bits, sendo os próprios estados iniciais dos registradores, os valores das sementes para a geração das sequiências. A precisão binária é de 16 e 4 bits para as partes fracionária e inteira, respectivamente. 


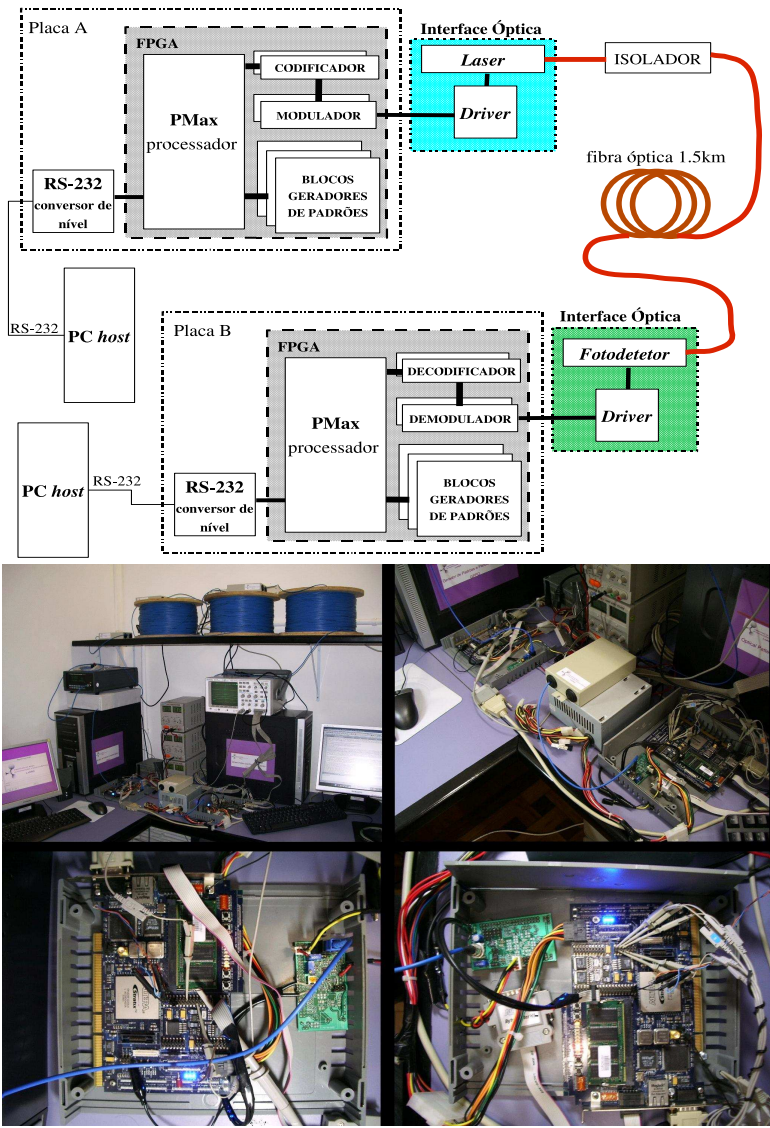

Fig. 2. Protótipo do Gerador de Padrões Ópticos (O2PG): a) Diagrama de Blocos b) Fotos.

Os parâmetros básicos para geração do padrão $S-\alpha-S$ são enviados do PC à placa FPGA através do barramento serial. As amostras com distribuição Uniforme são submetidas às transformações segundo as Eq.3 e Eq.4, seguidas das operações de escala e de deslocamento definidas na Eq.5.

\section{Protótipo do Gerador de PAdrões Ópticos}

Os experimentos da geração em hardware foram montados no protótipo do sistema O2PG (Optical Pattern and Packet Generator) do LaRSO (Laboratório de Comunicações e Sistemas Ópticos, http://larso.ime.eb.br). No O2PG já estão implementados os padrões Uniforme, Exponencial, Gaussiano e movimento Browniano fracionário. Em [6], foram apresentados os métodos de geração destes padrões e demonstrado a sua realização, com sucesso, nos domínios eletrônico e óptico. A Fig. 2 ilustra o esquemático e a montagem do protótipo do O2PG onde observa-se 2 estações PC, com 1 placa FPGA cada, onde é realizada a geração eletrônica dos diferentes padrões. As 2 estações estão conectadas por fibra óptica de 1,5 Km de extensão. As amostras geradas no meio eletrônico (FPGA) são posteriormente codificadas (bit-serial) e moduladas no comprimento de onda $1550,92 \mathrm{~nm}$ de uma fonte laser operando em freqüência de $622 \mathrm{MHz}\left(f_{0}\right)$. A freqüência máxima nominal da FPGA é de $150 \mathrm{MHz}$. Pulsos de luz foram gerados a cada $T$ segundos $\left(T=1 / f_{0}\right)$ e o sinal óptico é obtido segundo o padrão $S-\alpha-S$. A técnica FSK (frequencyshift keying) foi adotada considerando-se as freqüências 300
MHz e $600 \mathrm{MHz}$ para a modulação dos bits 0 e 1 , respectivamente. Para a modulação da fonte laser ou óptica, é utilizado um driver com controle automático de potência para alta velocidade e baixa voltagem (fonte $+3,3 \mathrm{~V} / 30 \mathrm{~mA}$ ). $\mathrm{O}$ controle de potência é utilizado para a garantia da intensidade do sinal óptico modulado. Um dispositivo isolador é aplicado para a garantia da integridade do laser. Após a geração, a transmissão e a detecção ópticas, os bits são re-convertidos para o meio eletrônico onde é realizada a validação do padrão e as medidas de desempenho pertinentes. Como a frequiência de geração eletrônica na FPGA é alta e igual a da fonte laser empregada, não foi necessária a realização de compressão óptica nos experimentos realizados neste trabalho.

\section{RESULTADOS EXPERIMENTAIS}

Os testes experimentais realizados compreendem a estimação dos parâmetros básicos do padrão $S-\alpha-S$ e a análise comparativa das curvas HTD. Os resultados obtidos pela geração em hardware foram confrontados com os obtidos com sequiências amostrais geradas em software (SW), programação $\mathrm{C}++$, de amostras segundo a família de fdps $S-\alpha-S$ apresentadas na seção II.B.

\section{A. Parâmetros de desempenho da implementação FPGA}

A periodicidade das seqüências é dada em função dos blocos URNG, cujo valor é de $2^{80}$ amostras por segundo ou 1 amostra por ciclo de clock. Na implementação FPGA foram consumidos cerca de 3.000 elementos lógicos do total de 25.000, além de 125 Kbytes de memória e 2 blocos de RAM. A frequiência máxima de clock atingida para o bloco $S-\alpha-S$ foi de $150 \mathrm{MHz}$.

\section{B. Estimação dos parâmetros $\alpha, \beta, \gamma e \mu$}

Conforme mencionado anteriormente, a estimação dos parâmetros de padrões $S-\alpha-S$ é um complexo desafio para as pesquisas da área de teoria de estimação. Em [13], os autores definiram o número de $\operatorname{amostras~}(n)$ necessário de forma a restringir a probabidade de erro de estimação como $n \geq 10^{k /(1-1 / \alpha)}$ sendo $k$ o número de casas decimais para a estimativa do parâmetro $\alpha$.

Os resultados da estimação dos parâmetros $\alpha(S-\alpha-S$ com $\beta=0$ ) obtidos com o método McCulloch, podem ser observados na Tab.I. Os demais parâmetros foram fixados em $\mu=0,010$ e $\gamma=0,0601$. Estes valores foram os mesmos adotados pelos autores em [12]. Geralmente, os parâmetros $\mu$ e $\gamma$ não diferem de seus valores originais após sua estimação sendo a impulsividade, o parâmetro mais crítico. Os valores HW1 e HW2 correspondem a estimação dos parâmetros após a geração eletrônica (FPGA) e após transmissão e recepção ópticas, respectivamente. Note que os parâmetros gerados são bem próximos aos obtidos pelas expressões da fdp da distribuição $S-\alpha-S$ (SW). Nos testes experimentais, não foram identificados erros durante a transmissão ópticas ou na recepção dos bits (foto-detector). Futuramente, seria interessante a inclusão de geradores de ruídos para a verificação do impacto do sistema de comunicação óptico nas amostras $S-\alpha-S$. 
TABELA I

Resultados Estimação Parâmetros Padrão $S-\alpha-S$

\begin{tabular}{|c|c|c|c|c|c|}
\hline Entrada & SW & HW1 & HW2 & Número de amostras & precisão \\
\hline 2,0 & 2,0 & 1,98 & 1,99 & 10.000 & $\pm 0,01$ \\
\hline 1,7 & 1,72 & 1,71 & 1,71 & 72.000 & $\pm 0,01$ \\
\hline 1,5 & 1,51 & 1,49 & 1,5 & 128.000 & $\pm 0,02$ \\
\hline
\end{tabular}

\section{Curvas HTD}

As curvas HTD obtidas para as seqüências de amostras com padrão $S$ - $\alpha$ - $S$ e indíces de impulsividade $\alpha=1,7$ e $\alpha=1,5$ estão apresentadas nas Figs 3 e 4. Pode-se observar a cauda-

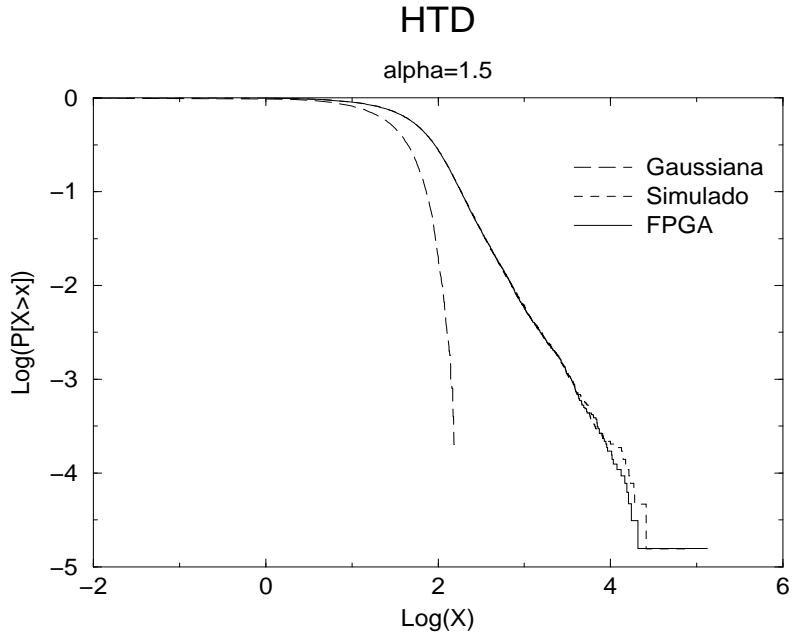

Fig. 3. Curvas HTD para $\alpha=1,5$ e $\alpha=2,0$.

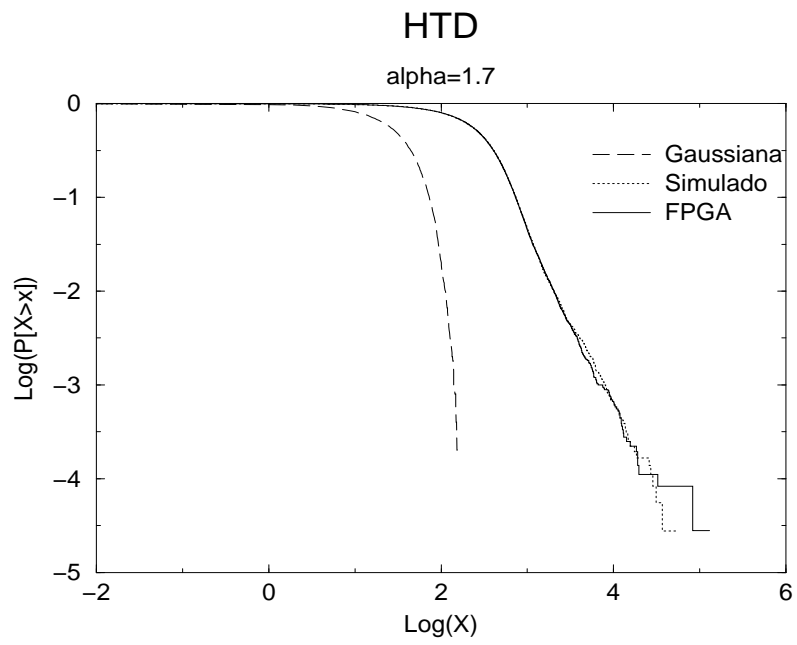

Fig. 4. Curvas HTD para $\alpha=1,7$ e $\alpha=2,0$.

pesada ou mais longa dos processos com $\alpha=1,7$ e $\alpha=1,5$ em comparação a distribuição Gaussiana $(\alpha=2)$. Nota-se também que as curvas HTD, obtidas pelas amostras geradas pela FPGA, são bem semelhantes as curvas obtidas em SW. Assim, demonstra-se que as transformações realizadas para a geração do padrão $S-\alpha-S$ em hardware tiveram sucesso e se aproximam das fdps (teóricas) definidas na seção II.B.

\section{CONCLUSÕES}

Este trabalho apresentou um novo método de geração de sinais ópticos com padrões baseado na família de distribuições $\alpha$-stable. O gerador foi implementado e testado em arquitetura FPGA. Experimentos foram realizados em um ambiente com transmissão por fibra óptica com $1,5 \mathrm{Km}$. Os resultados demonstraram a eficácia do gerador em termos de representação dos parâmetros básicos e da distribuição de cauda-pesada. Devido a melhor representação de sinais reais e com diferentes índices de impulsividade, o gerador de padrões $\alpha-S$ proposto demonstra ser bastante promissor para os testes dos futuros sistemas de comunicações ópticos.

\section{REFERÊNCIAS}

[1] G. Agrawal, Fiber-Optic Communication Systems. USA: John-Wiley and Sons, 2002.

[2] G. Papadimitriou, C. Papazoglu, and A. Pomportsis, "Optical switching: Switch fabrics, techniques and architectures," IEEE Journal of Lightwave Tecnology, vol. 21, pp. 384-405, February 2003.

[3] S. Yao, B. Mukherjee, and S. Dixit, "Advances in photonics packet switching: An overview," IEEE Comm. Magazine, vol. 38, pp. 84-94, February 2000.

[4] N. Cheung, "Fiber to 30 million homes," IEEE Communications Magazine, vol. 43, pp. 70-71, September 2005 .

[5] D. Lee, J. Villasenor, W. Luk, and P. Leong, "A hardware gaussian noise generator using the box-müller method and its error analysis," IEEE Trans. on Computers, vol. 6, pp. 659-671, June 2006.

[6] G. Loss and R. Coelho, "Fpga-based fractional brownian motion signalpatterns for optical packet generation," Proceedings of the IEEE/ITS 2006, September 2006.

[7] M. Box and M. Müller, "A note on the generation of random normal deviates," Annals Math. Statistics, vol. 29, pp. 610-611, 1958.

[8] M. Shao and C. Nikias, "Signal processing with fractional lower order moments: Stable processes and their applications," Proceedings of the IEEE, vol. 81, no. 7, pp. 986-1010, 1993.

[9] C. Nikias and M. Shao, Signal Processing with Alpha-Stable Distributions and Applications. New York: Wiley, 1995.

[10] P. Georgiou, P. Tsakalides, and C. Kyriakakis, "Alpha-stable modeling noise and robust time-delay estimation in the presence of impulsive noise," IEEE Trans. on Multimedia, vol. 1, pp. 291-301, September 1999.

[11] G. Samorodnitsky and M. Taqqu, Stable Non-Gaussian Random Processes: Stochastic Models and Infinite Variance. London: Chapman \& Hall, 1994.

[12] S. Bates and S. McLaughlin, "The estimation of stable distribution parameters from teletraffic data," IEEE Transactions on Signal Processing, vol. 48, pp. 865-870, March 2000.

[13] M. Crovella and L. Lipsky, Simulations with Heavy-Tailed Workloads: Self-similar Network Traffic and Performance Evaluation: Chapter 3. John Wiley and Sons, 2000.

[14] P. Lévy, "Cacul des probabilités," Paris: Gauthier-Villars, pp. 13601366, 1925.

[15] H. McCulloch, "Simple consistent estimators of stable distribution parameters," Communications in Statistics, vol. 15, no. 4, pp. 11091136, 1998.

[16] S. kogon and D. Williams, "On the characterization of impulsive noise with $\alpha$-stable distributions using fourier techniques," 29th Asilomar Conference Signals, Syst. and Comp., 1995.

[17] X. Ma and L. Nikias, "Parameter estimation and blind channel identification of impulsive signal environments," IEEE Trans. on Signal Processing, vol. 43, pp. 2884-2897, 1995.

[18] G. Tsihrintzis and L. Nikias, "Fast estimation of parameters of alphastable impulsive interference," IEEE Trans. on Signal Processing, vol. 44, pp. 1492-1503, 1996.

[19] J. Nolan, Stable Distributions: Models for Heavy Tailed Data. American University Press, 2005.

[20] T. Tkacik, "A hardware random number generator," 4th International Workshop on Cryptographic Hardware and Embedded Systems, vol. 1, pp. 450-453, August 2002. 